\title{
Who contributes? A strategic approach to a European immigration policy
}

\author{
Giuseppe Russo ${ }^{1,2^{*}}$ and Luigi Senatore $e^{3,4}$
}

\footnotetext{
*Correspondence: grusso@unisa.it 1 Dipartimento di Scienze

Economiche, Università di Salerno, via Ponte don Melillo, Fisciano (SA), Italy

${ }^{2}$ CSEF, Napoli, Italy

Full list of author information is available at the end of the article
}

\begin{abstract}
Who pays for immigration control? According to the Lisbon Treaty the cost of enforcing the European border against illegal immigration shall be shared among the EU members. Nonetheless, the Treaty is vague with respect to the "appropriate measures" to adopt in order to distribute the financial burden. Members who do not share their borders with source countries have an incentive to free ride on the other countries. We study a novel contribution game in which a border country and a central country minimize a loss function with respect to their national immigration target. We consider both sequential and simultaneous decisions and we show that joint contribution occurs only if the immigration targets are not too different. Total contribution is higher in the simultaneous game, but the sequential framework reduces the incentive to free ride.
\end{abstract}

Jel codes: $\mathrm{D} 78, \mathrm{H} 72, \mathrm{H} 77$

Keywords: Policy making; Government expenditures; Local government expenditures; Federalism

\section{Introduction}

The Amsterdam Treaty in 1997 and the Tampere Meeting in 1999 have been early attempts to lay out the foundations of a common EU immigration and asylum policy.

More recently, the Lisbon Treaty calls for the fair sharing of the financial burden necessary for border control and for the development of a European border surveillance system (Eurosur) (articles no. 77 and 80).

In spite of these aspirations, results have been deceiving: member states still control the most important aspects of immigration policy, and the Constitutional Treaty reiterates the right of the EU members to determine national immigration quotas (article III-267 (5)). ${ }^{1}$

So far, the main attempt to move immigration control to a supranational level has been the establishment of the Frontex agency in 2005. The intent of Frontex is coordinating the national immigration policies at the European level, but it is still far from being a full-fledged authority.

The promotion of a EU immigration policy, the development of Eurosur and the expansion of Frontex will require important financial resources (European Commission 2008; Jeandesboz 2008).

However, although the Lisbon Treaty states that the cost of implementing UE policies "shall be governed by the principle of solidarity and fair sharing of responsibility between

(c) 2013 Russo and Senatore; licensee Springer. This is an Open Access article distributed under the terms of the Creative

Commons Attribution License (http://creativecommons.org/licenses/by/2.0), which permits unrestricted use, distribution, and reproduction in any medium, provided the original work is properly cited. 
the Member States" 2 , it does not provide any rule on how to share these costs in practice, nor does it regulate the institutional framework in which the funds have to be gathered.

According to Schain (2010), this is the key indication of the failure of immigration policy to take off at the European level.

This paper studies to what extent it is possible to implement a EU immigration policy when the member states interact in the absence of a regulator.

The literature on this issue is still in its beginning, even though authors concerned with the analysis of asylum policies have addressed similar problems: they have stressed the cross-country spillovers caused by refugees entitled to free mobility within a federation, and they have studied the welfare effects of a coordinated policy. This literature is in fact particularly interested in studying the gains from coordination. For instance, Hatton (2004) adopts a standard social planner approach, while Facchini et al. (2006) study the welfare properties of policy coordination in a representative democracy framework.

However, we move from a political economy perspective, and our work is mainly related to two contributions: Mayr et al. (2011) and Haake et al. (2010). The first article studies the joint funding of immigration restriction when a border country may legalize illegal immigrants who are then entitled to move to an interior country. Still, the analysis is limited to the consequences of an immigration amnesty.

The second article proposes a mechanism-design approach in order to redistribute resources for immigration control from northern countries to southern countries ${ }^{3}$. The tools provided by mechanism design might be useful in this respect, but the lack of a federal regulator allowed to tax and redistribute suggests that it is still too early to put forward such an approach ${ }^{4}$.

In what follows we investigate the possibility of sharing the cost of external border control in the framework of the current EU institutions. The simplest method for trying to achieve this aim would be transferring EU funds to the countries that lie on the external border of the Union.

In this respect, two crucial points must be emphasized: 1) there is no federal regulator; 2) free mobility in the Schengen area implies that countries enforcing the external border -i.e. Southern European countries- provide a public good. ${ }^{5}$

These features are typical of contribution games, which therefore provide a proper modelling framework for our purposes. ${ }^{6}$

However, immigration policy has a peculiarity that deserves special attention: immigration is restricted because the potential supply of foreign workers is overwhelming, and different countries have different preferences over the number of immigrants to be let in. In other words, countries have a national immigration target.

Several authors have studied the determination of this target in various theoretical frameworks. To mention but a few, the seminal paper by Benhabib (1996) is an example of median voter models. Facchini and Willmann (2005) adopt a common agency approach. Mazza and Van Winden (1996), Amegashie (2004), Epstein and Nitzan (2006), Russo (2011), determine immigration quotas from voting, lobbying and/or bargaining models.

What do these targets imply for the funding of immigration control in a federation? Not only this issue has not yet been investigated, but it also requires the development of a specific contribution game, since the possibility of a target has not been explored in the theoretical literature. We build such a game by observing that deviations from a target 
cause disutility. We capture this point by introducing loss functions in a contribution game.

In order to clarify our approach, it is important to note that we want to depict a situation in which national governments try to achieve their own interests in a federal assembly. For our purposes, therefore, the national targets are exogenously given. ${ }^{7}$

Our model includes a central country (henceforth $C$ ) and a border country (henceforth $B$ ). $B$ shares its border with an emigration country and provides border enforcement for both countries. Both $C$ and $B$ should contribute to border control, but they have different immigration targets and contribution cannot be enforced. We believe that these features fit quite well the current EU situation.

Our procedure brings to light several important results. First of all, we find that the difference in the national targets is crucial, and it could easily prevent contribution although information is complete and symmetric. Thus, imperfect information is not the main obstacle to a European immigration policy.

Then, we compare simultaneous and sequential decisions to check whether there exists an institutional framework that dominates the others in terms of total contribution or incentive to contribute. In the sequential case we explore what happens when the leader is $C$ or $B .^{8}$

By confronting the alternative regimes we find that:

1) in order to obtain positive contributions, the immigration targets of $C$ and $B$ must not be too different;

2) the admissible difference in the immigration targets is wider in the sequential game;

3) when both contributions are positive, total contribution is unambiguously higher in the simultaneous game;

4) equilibrium contributions are Pareto-inefficient;

5) in each game a simple condition determines whether $C$ or $B$ contributes more.

Results 1) and 2) reverse the well-known conclusions in Varian (1994): they show that the presence of a target incentivizes contribution when preferences are similar and that free riding is less likely in the sequential game.

With respect to the prospects of a European immigration policy at the current stage of the integration process, our results suggest that a simultaneous regime, in which central countries decide jointly with border countries, produces tighter border enforcement but is more vulnerable to free riding. On the other hand, the sequential regime provides better incentives to contribute in spite of a smaller total contribution.

The paper is organized as follows: the next Section introduces our model, Section 3 presents the results when decisions are sequential or simultaneous, Section 4 studies the effect of the cost asymmetry on the equilibrium contribution, Section 5 is devoted to compare the equilibrium contributions under the different institutional frameworks, Section 6 proves that the equilibrium contributions are Pareto inefficient, and Section 7 concludes.

\section{The model}

Our model has to depict the basic issues related to the European immigration policy we have discussed in the introduction. First of all, external border enforcement is a public good, and there exists a conflict over its funding. No supranational authority can enforce 
a scheme of taxes and subsidies, thus countries interact strategically and nobody is forced to contribute. As a consequence, a contribution game fits very well this situation.

Our players are a central country $(C)$ and a border country $(B) . B$ shares its border with an emigration country and provides immigration control for $C$ as well. Both countries can contribute to curb immigration, but they have different immigration targets and face possibly different costs of contributing.

Before introducing our contribution game we have to clarify how immigration control is obtained.

\subsection{Immigration control}

Each country would like to halt immigration once its target is achieved. However, restricting immigration is expensive: it requires resources to enforce the border, screen immigrants, contrast illegal inflows and so on. A convenient way to summarize these actions is describing immigration restriction as an output produced through the resources $C$ and $B$ are willing to spend in order to achieve their targets.

We define with $g_{B}$ and $g_{C}$ the contributions by $B$ and $C$ respectively. Let $M$ be the inflow of immigrants into the federation, which is given by

$$
M=\bar{M}-d\left(g_{B}+g_{C}\right) \quad 0<d<1 ;
$$

Where $\bar{M}>0$ depicts the inflow into the federation in case of no restriction (i. e. $g_{B}=$ $g_{C}=0$ ). This kind of "production function" fits the idea that the amount of restriction is proportional to the resources used. ${ }^{9}$

\subsection{Payoffs}

As we have pointed out in the introduction, the peculiarity of immigration control is that the country's desired quota acts as a bliss point. Thus, payoffs include a quadratic loss function with respect to the national target, given by $0 \leq M_{C}^{*}<\bar{M}$ and $0 \leq M_{B}^{*}<\bar{M}$ respectively.

Since we are interested in studying how equilibrium contributions are determined, we consider the targets as exogenously given. In our view, this fits well a situation in which member countries try to defend their national interests within a federal assembly like the European Parliament.

We also assume perfect information on the destination chosen by immigrants: both countries know how many immigrants are willing to settle in $C$ and how many immigrants are willing to settle in $B$. This assumption is by no means restrictive: since mobility within the federation is unrestrained, it only indicates that $C$ and $B$ know how the population inflow is going to be shared. Countries are usually well informed about their relative attractiveness for the immigrants.

Finally, $C$ and $B$ bear a quadratic cost to collect the resources needed to enforce the border. ${ }^{10}$ As a consequence, we write the utilities as follows:

$$
\begin{aligned}
& U_{C}=-\frac{1}{2}\left(M-M_{C}^{*}\right)^{2}-\frac{1}{2} g_{C}^{2} \\
& U_{B}=-\frac{1}{2}\left(M-M_{B}^{*}\right)^{2}-\frac{\pi}{2} g_{B}^{2} .
\end{aligned}
$$

Note that since $M$ is the inflow into the federation, $M_{C}^{*}$ and $M_{B}^{*}$ denote the federal target preferred by $\mathrm{C}$ and $\mathrm{B}$ respectively. ${ }^{11}$ 
The parameter $\pi \geq 1$ allows for the possibility that the disutility of gathering the resources needed to curb immigration is relatively higher for $\mathrm{B}$.

$\pi$ is introduced for sake of generality, because it gives us the opportunity to analyze a cost asymmetry: in principle, it is quite possible that $\mathrm{C}$ and $\mathrm{B}$ bear different costs to gather the same contribution. This might happen, for instance, when B faces a fiscal crisis, as it is currently the case of Italy and Spain.

By substituting (1) into (2) and (3) we can rewrite the payoffs:

$$
\begin{aligned}
& U_{C}=-\frac{1}{2}\left(\bar{M}-d\left(g_{B}+g_{C}\right)-M_{C}^{*}\right)^{2}-\frac{1}{2} g_{C}^{2} \\
& U_{B}=-\frac{1}{2}\left(\bar{M}-d\left(g_{B}+g_{C}\right)-M_{B}^{*}\right)^{2}-\frac{\pi}{2} g_{B}^{2}
\end{aligned}
$$

We are now going to solve the model under sequential and simultaneous decisions. In order to avoid redundancies the main properties of the results are discussed at the end of this section.

\subsection{Results: sequential decisions}

In the case of sequential decisions, both $C$ and $B$ could have the right to move first. We are now going to explore both cases.

\subsubsection{C moves first}

Assume for the moment that $C$ is the leader and $B$ is the follower. We solve the game by backwards induction. The best response of $B$ to $C$ is

$$
\bar{g}_{B}=\frac{d\left(\bar{M}-M_{B}^{*}\right)-d^{2} g_{C}}{\pi+d^{2}} .
$$

By substituting (6) into (4) we can write the leader's problem:

$$
\max _{g_{C}} U_{C}=-\frac{1}{2}\left[\bar{M}-d\left(g_{C}+\frac{d\left(\bar{M}-M_{B}^{*}\right)-d^{2} g_{C}}{\pi+d^{2}}\right)-M_{C}^{*}\right]^{2}-\frac{1}{2} g_{C}^{2}
$$

which yields

$$
g_{C}^{*}=\frac{\Delta_{C}\left(\pi+d^{2}\right) \pi d-\pi d^{3} \Delta_{B}}{\pi^{2} d^{2}+\left(\pi+d^{2}\right)^{2}}
$$

where $\Delta_{C} \equiv\left(\bar{M}-M_{C}^{*}\right)$, and $\Delta_{B} \equiv\left(\bar{M}-M_{B}^{*}\right)$ measure the desired entry restriction.

By substituting (7) into (6) we get

$$
g_{B}^{*}=\frac{\Delta_{B}\left(\pi+d^{2}+\pi d^{2}\right) d-\pi d^{3} \Delta_{C}}{\pi^{2} d^{2}+\left(\pi+d^{2}\right)^{2}}
$$

we therefore have obtained the equilibrium contributions of both players when $C$ moves first.

These contributions are positive under the following conditions:

$$
\begin{aligned}
& g_{C}^{*}>0 \text { for } \frac{\Delta_{C}}{\Delta_{B}}>\frac{d^{2}}{\pi+d^{2}} \\
& g_{B}^{*}>0 \text { for } \frac{\Delta_{C}}{\Delta_{B}}<\frac{\pi+d^{2}+\pi d^{2}}{\pi d^{2}}
\end{aligned}
$$

Since $\Delta_{C}$ and $\Delta_{B}$ measure the restriction desired by $C$ and $B$ respectively, we define the ratio $\frac{\Delta_{C}}{\Delta_{B}}$ as the "relative restriction" desired by $C$. 
$\frac{\Delta_{C}}{\Delta_{B}}>1$ means that $C$ likes more restriction relative to $B$. The opposite occurs when $\frac{\Delta_{C}}{\Delta_{B}}<1$. Conditions (9) and (10) indicate that for a player to contribute positively his desired relative restriction must be sufficiently high. This will be crucial in the rest of the paper.

Now we are going to present the results when $B$ is the leader.

\subsubsection{C moves second}

When $B$ moves first, the best response function of $C$ is

$$
\bar{g}_{C}=\frac{d\left(\bar{M}-M_{C}^{*}\right)-d^{2} g_{B}}{1+d^{2}} .
$$

In order to solve the leader's problem, we now substitute the best response function of $C$ (11) into (5) and we find the equilibrium contribution of $B\left(g_{B}^{* *}\right)$. Then, we plug $g_{B}^{* *}$ into (11) and we solve for the follower's contribution $\left(g_{C}^{* *}\right)$.

The equilibrium contributions are

$$
\begin{aligned}
& g_{C}^{* *}=\frac{\Delta_{C}\left(d^{2}+\pi+\pi d^{2}\right) d-d^{3} \Delta_{B}}{d^{2}+\pi\left(1+d^{2}\right)^{2}} \\
& g_{B}^{* *}=\frac{\Delta_{B}\left(1+d^{2}\right) d-d^{3} \Delta_{C}}{d^{2}+\pi\left(1+d^{2}\right)^{2}} .
\end{aligned}
$$

The conditions for having positive contributions are summarized below:

$$
\begin{aligned}
& g_{C}^{* *}>0 \text { for } \frac{\Delta_{C}}{\Delta_{B}}>\frac{d^{2}}{d^{2}+\pi+\pi d^{2}} \\
& g_{B}^{* *}>0 \text { for } \frac{\Delta_{C}}{\Delta_{B}}<\frac{1+d^{2}}{d^{2}} .
\end{aligned}
$$

Finally, we are going to solve the simultaneous game.

\subsection{Results: simultaneous decisions}

In a simultaneous game, the best response functions for $C$ and $B$ are, respectively, (11) and (6), and the solutions are

$$
\begin{gathered}
\tilde{g}_{C}=\frac{\Delta_{C}\left(\pi+d^{2}\right) d-d^{3} \Delta_{B}}{d^{2}+\pi+\pi d^{2}} \\
\tilde{g}_{B}=\frac{\Delta_{B}\left(1+d^{2}\right) d-d^{3} \Delta_{C}}{d^{2}+\pi+\pi d^{2}} .
\end{gathered}
$$

These contributions are positive under the following conditions:

$$
\begin{array}{cc}
\tilde{g}_{C}>0 \text { for } & \frac{\Delta_{C}}{\Delta_{B}}>\frac{d^{2}}{d^{2}+\pi} \\
\tilde{g}_{B}>0 \text { for } & \frac{\Delta_{C}}{\Delta_{B}}<\frac{1+d^{2}}{d^{2}}
\end{array}
$$

By observing (7), (8), (12), (13), (16) and (17) it is evident that the equilibrium contribution of each player is decreasing with respect to the desired immigration restriction of the other player. In other words, in all cases the contribution of $C$ is decreasing with $\Delta_{B}$, and the contribution of $B$ is decreasing with $\Delta_{C}$.

To understand intuitively this result, suppose then that $B$ prefers strict border enforcement and $C$ is relatively open. As a consequence the ratio $\frac{\Delta_{C}}{\Delta_{B}}$ is low, and $C$ has an incentive to free ride, because $B$ will provide enough immigration control for both countries. This 
conveys the essential insight that, in order for both countries to contribute, the national $\operatorname{targets} M_{C}^{*}$ and $M_{B}^{*}$ must not be too different. This result extends earlier findings by Varian $(1994)^{12}$, and it has crucial consequences that we are going to discuss in the rest of the paper.

Before proceeding to compare the outcomes under the sequential and the simultaneous regimes, it is indispensable to understand when contributions are positive and when there exists joint contribution.

\section{Conditions for joint contribution}

We define "joint contribution" a situation in which both contributions are positive in equilibrium. We know that individual equilibrium contributions are positive when conditions (9), (10), (14), (15), (18) and (19) hold. The cut-off values of $\frac{\Delta_{C}}{\Delta_{B}}$ are ordered in Figure 1, and the intervals of $\frac{\Delta_{C}}{\Delta_{B}}$ under which both contributions are positive in the different games are denoted by lines in red and bold.

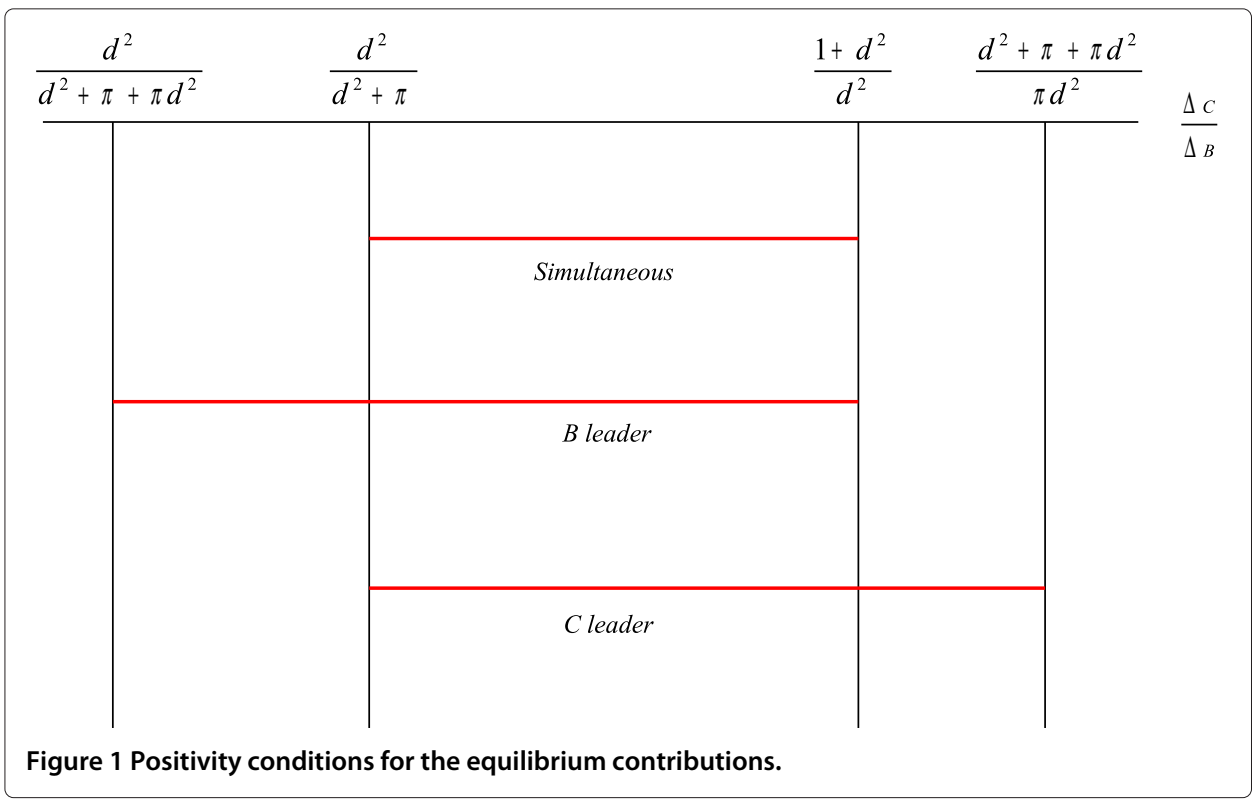

By simple inspection of these conditions we can write the following proposition:

Proposition 1. (Conditions for joint contribution): joint contribution occurs if and only if the individual immigration targets are not too different. The admissible difference is broader in the sequential game.

Proof. See the Appendix.

The proposition is crucial because it points out that in a sequential framework the range of $\frac{\Delta_{C}}{\Delta_{B}}$ under which there exists joint contribution is wider compared to the simultaneous framework (see Figure 1). In this respect, our results depart from Varian (1994), who argues that sequentiality can exacerbate free riding problems: in Varian a leader with 
higher marginal utility from the public good might be better off by not contributing and free riding on the follower.

In our model this result is reversed because a player does not contribute only when the other player's contribution is sufficient to saturate his utility. This occurs only when the players have very different targets.

In a sequential game with close targets the follower's contribution is not sufficient to put the leader on his bliss point, so the leader has no incentive to free ride.

As a consequence, the only way to exploit the leadership is trying to set the contribution at a level that does not satisfy the follower and pushes him to add his own contribution.

\section{The role of the cost asymmetry}

In this section we report some comparative statics results with respect to the effect of the cost asymmetry $\pi$. For simplicity we only show the case in which $B$ bears higher costs. The results are obviously symmetric when $C$ bears higher costs.

In the Appendix we show that, quite intuitively, $B$ reduces his equilibrium contribution as $\pi$ increases. On the other hand, the equilibrium contribution of $C$ increases with $\pi$ in all cases, provided that joint contribution occurs. Results are summarized in the following table:

sequential

$$
\begin{aligned}
& C \text { leader } \\
& \frac{\partial g_{C}^{*}}{\partial \pi}>0 \text {; } \\
& \begin{array}{c}
\text { B leader } \\
\frac{\partial g_{C}^{* *}}{\partial \pi}>0 \text { for } \frac{\Delta_{C}}{\Delta_{B}}<\frac{1+d^{2}}{d^{2}}
\end{array} \\
& \frac{\partial g_{B}^{*}}{\partial \pi}<0 \\
& \frac{\partial g_{B}^{* *}}{\partial \pi}<0 \\
& \frac{\partial \tilde{g}_{C}}{\partial \pi}>0 \text { for } \quad \frac{\Delta_{C}}{\Delta_{B}}<\frac{1+d^{2}}{d^{2}} \quad \frac{\partial \tilde{g}_{B}}{\partial \pi}<0 \text { for } \frac{\Delta_{C}}{\Delta_{B}}<\frac{1+d^{2}}{d^{2}}
\end{aligned}
$$

The most important outcome of this comparative statics analysis is that when there is joint contribution the timing of the game does not determine the effect of $\pi$ on the equilibrium contributions.

What matters is the decision to contribute: once $C$ decides to put resources in immigration control, he is going to increase his equilibrium contribution as $B$ faces higher costs in gathering his own contribution. To understand the reason of this behavior it is important to remember that this holds when both contributions are positive, i.e. when the targets of $C$ and $B$ are sufficiently close. In such a case, $C$ finds it convenient to increase his equilibrium contribution in order to compensate the possible disadvantage of $B$.

\section{Sequential vs. simultaneous decisions}

\subsection{Total contribution}

In this section we restrict our attention to the case of joint contribution. By comparing the equilibrium solution in the three cases, it is straightforward to conclude that total contribution is higher in the simultaneous regime. This is summarized in the following proposition:

Proposition 2. (Comparison of total contributions): when joint contribution occurs, total contribution is higher in the simultaneous game than in the sequential one. 
Proof. See the Appendix.

The proposition simply states that the simultaneous game dominates the sequential game in terms of total contribution -no matter who is the leader-. This happens because when the moves are simultaneous there is no first-mover advantage, and the leader cannot push the follower to contribute in its stead. Unlike Proposition 1, this result is in line with Varian (1994), who shows that in a game with complete information total contribution is never larger in the sequential framework. ${ }^{13}$

Proposition 1 and Proposition 2 convey our most important result, namely that the simultaneous game increases total contribution, but it requires more stringent conditions in order to get positive contributions from both players.

In other words, the simultaneous framework is successful in increasing total contribution given that countries are willing to contribute, while the sequential framework is successful in inducing contribution. It follows that the sequential game should be recommended when the immigration targets of $C$ and $B$ are very different and the main issue is to provide an incentive to contribute. This seems to be the case of the EU, therefore an effort to frame a federal immigration policy at the current stage of the European integration should favor sequential funding decisions.

In addition, we must stress that the simplest attempt to obtain some contribution from a reluctant country is to make it act as a follower in the sequential game. In fact, from Proposition 1 we know that the leader tries to set his own contribution at a level that encourages the follower to contribute as well. This widens the range of $\frac{\Delta_{C}}{\Delta_{B}}$ allowing a positive contribution (see Figure 1).

\subsection{Individual contribution}

We now compare the individual contributions within the different regimes in the case of joint contribution.

Our first conclusion is summarized in the following proposition:

Proposition 3. (Equilibrium contributions in the sequential game): in the sequential game the leader contributes more than the follower when $\frac{\Delta_{C}}{\Delta_{B}}>\frac{\pi+d^{2}+2 \pi d^{2}}{\pi+2 \pi d^{2}}$ (C leader) and when $\frac{\Delta_{C}}{\Delta_{B}}<\frac{1+2 d^{2}}{\pi+\pi d^{2}+2 d^{2}}$ (B leader).

Proof. See the Appendix.

To understand the meaning of this proposition, consider the case of $C$ leader, and notice that the cut-off value of $\frac{\Delta_{C}}{\Delta_{B}}$ is $\frac{\pi+d^{2}+2 \pi d^{2}}{\pi+2 \pi d^{2}}>1$. This means that for the leader to contribute more than the follower his desired relative restriction must be sufficiently high ${ }^{14}$.

This happens because he exploits the information on the follower's target. Thus in our game the first mover advantage has two aspects: 1) the leader can push the follower to contribute (proposition 1); 2) the leader can reduce his own contribution as the follower has a stronger taste for restriction.

The comparison of the individual contributions in the simultaneous game is reported in the next proposition: 
Proposition 4. (Equilibrium contributions in the simultaneous game): in the simultaneous game C contributes more than $B$ if $\frac{\Delta_{C}}{\Delta_{B}}>\frac{1+2 d^{2}}{\pi+2 d^{2}}$.

Proof. See the Appendix.

To understand intuitively the meaning of this proposition, notice that when costs are symmetric (i.e. $\pi=1$ ) the condition $\frac{\Delta_{C}}{\Delta_{B}}>\frac{1+2 d^{2}}{\pi+2 d^{2}}$ boils down to $\Delta_{C}>\Delta_{B}$. Hence, when the cost of gathering the resources for immigration control is the same, the country who desires more restriction contributes more. When $\pi$ is larger than unity this condition is relaxed: we have $\tilde{g}_{C}>\tilde{g}_{B}$ if $\Delta_{C}>\left(\frac{1+2 d^{2}}{\pi+2 d^{2}}\right) \Delta_{B}$, with $\left(\frac{1+2 d^{2}}{\pi+2 d^{2}}\right)<1$.

In other words, $C$ observes that $B$ bears a higher cost, and, if $\pi$ is sufficiently high, $C$ is going to contribute more than $B$ even though $\Delta_{C}<\Delta_{B}{ }^{15}$.

\section{Efficiency}

We now address the issue of Pareto Efficiency. We define the social welfare $W$ as the weighted sum of the utilities (2) and (3):

$$
W=-\frac{\alpha}{2}\left[\left(M-M_{C}^{*}\right)^{2}+g_{C}^{2}\right]-\frac{(1-\alpha)}{2}\left[\left(M-M_{B}^{*}\right)^{2}+\pi g_{B}^{2}\right],
$$

where $0<\alpha<1$ and $(1-\alpha)$ are the weights that the social planner assigns to $C$ and $B$ respectively. The weights may mirror, for instance, the relative size of the two countries in the federation. Since the outcome is clearly inefficient when a player free rides, we focus our attention on the case of joint contribution. Then, it is straightforward to prove the following proposition:

Proposition 5. (Pareto efficiency): the equilibrium contributions are efficient if and only if $(1-\alpha) \pi g_{B}^{*}=\alpha g_{C}^{*}$.

Proof. See the Appendix.

According to this condition, the marginal cost borne by each country should be weighted for its weight in the federation. Inefficiency arises when a planner can increase the social welfare by reallocating some contribution towards the country with the lower social marginal cost.

The efficiency condition can also be written as $\frac{\pi g_{B}^{*}}{g_{C}^{*}}=\frac{\alpha}{1-\alpha}$. Since the players do not internalize the social weights $\alpha$ and $(1-\alpha)$, there is no reason why in the decentralized equilibrium the ratio of the marginal costs should equal $\frac{\alpha}{1-\alpha}$. Thus, we conclude that the decentralized equilibrium is generally Pareto inefficient.

\section{Conclusions}

The strikingly simple model we have developed has novel and unexpected implications for framing a European immigration policy.

First of all, we bring to light that joint contribution occurs only if the national immigration targets are not too different. Therefore, the real root of the free riding problem lies in the heterogeneity of the national targets rather than in imperfect information. This is even more worrying because it implies that improving information will not help to avoid free riding. 
On the other hand, in contrast to Varian (1994), we uncover that the free riding problem is reduced in a sequential framework. Thus, when the federation members are heterogeneous and free riding is pervasive, sequential decisions should be definitely preferred. Since this case seems closer to the current EU situation, our model suggests to adopt a sequential contribution scheme in which border countries are the leader.

In addition, according to our results, once joint contribution is achieved central countries compensate to some extent the possible lack of resources of border countries. ${ }^{16}$ This fosters the idea that securing joint contribution should be the top priority for EU policymakers.

Thus, in the wait for a full-fledged federal immigration authority, our analysis provides a feasible roadmap to implement to some extent article no. 80 of the Lisbon Treaty.

\section{Endnotes}

${ }^{1}$ The emigration wave due to the Arab Spring has produced pressures to reintroduce internal border checks rather than promoting a European immigration policy. In April 2011 French President Nicolas Sarkozy and Italian Prime Minister Silvio Berlusconi sent a joint letter to the European Commission and the European Council, requesting EU to "review the possibility of temporarily restoring controls at international borders" in the Schengen area.

${ }^{2}$ Article no. 80.

${ }^{3}$ Haake et al. (2010) propose the adoption of the expected externality mechanism, where a supranational authority asks each country its own marginal willingness to pay for the public good, then countries are taxed and provided with the public good according to the revealed information. Unfortunately, this mechanism does not always satisfy the participation constraints.

${ }^{4}$ In addition, mechanisms are especially used to deal with informational asymmetries (see Clarke, 1971; Arrow, 1979; d'Aspremont and Gerard-Varet, 1979) while contribution games show that free riding can occur under perfect information.

${ }^{5}$ Northern members of the EU seem indeed reluctant to contribute to enforce the border in the South (Wolff, 2008).

${ }^{6}$ Note also that Frontex is funded through a subsidy of the EU plus "a contribution from the countries associated with the implementation, application and development of the Schengen acquis" and "any voluntary contribution from the Member States" (Official Journal of the EU, 25-11-2004, L 349/9).

${ }^{7}$ According to the literature quoted above, the target is determined by the interaction of different interest groups rather than by a benevolent social planner. As a consequence, it should not be intended as a social optimum. Deviations are costly because they represent a diversion from the national target, no matter how it has been determined.

${ }^{8} \mathrm{~A}$ great deal of literature studies joint provision of public goods within a sequential or simultaneous game (see for example Warr, 1982 and 1983; Cornes and Sandler, 1984; Bergstrom et al., 1986; Varian, 1994). With respect to the funding of border control, Mayr et al. (2011) only consider simultaneous decisions. They do not study what happens when a country can exploit the advantage of being the first mover.

${ }^{9}$ Linearity of eq. (1) is useful in order to get straightforward closed-form solutions. We have tested the robustness of our model by using a strictly concave form of (1). In such a case, when closed-form solutions are still available, the results reported in Proposition 
1 continue to hold in spite of substantial complications. In the absence of closed-form solutions, it is easy to get similar outcomes from numerical simulations. This supports the intuition that our results are due to the presence of a target rather than to the functional form of (1), thus we have preferred to preserve simplicity.

${ }^{10}$ Gathering real resources always generates costs: they can be the political costs of raising taxes, or even the opportunity costs of diverting funds from alternative projects.

${ }^{11}$ This is not an issue because free mobility and perfect information on the destination chosen by the immigrants imply that any national target uniquely defines a federal target. An example clarifies this point: suppose that one half immigrants settle in $C$ and the other half settle in $\mathrm{B}$. If $\mathrm{C}$ wants 100 immigrants, it follows that 200 should be admitted into the federation, thus its target will be $M_{C}^{*}=200$.

${ }^{12}$ Russo and Senatore (2012) provide the theoretical framework of a contribution game with loss functions and a thorough comparison with Varian (1994). They suggest several cases that involve the joint production of a public good in the presence of targets.

${ }^{13}$ We also have $\left(g_{C}^{*}+g_{L}^{*}\right) \geq\left(g_{C}^{* *}+g_{L}^{* *}\right)$ when $\frac{\Delta_{C}}{\Delta_{L}} \leq \frac{\pi\left(1+d^{2}\right)+d^{2}\left(2+d^{2}\right)}{\pi\left(1+2 d^{2}\right)+d^{2}\left(1+d^{2}\right)}$. There are no particular reasons why this condition should hold, thus we conclude that it is not possible to know a priori whether total contribution is higher when $C$ or $L$ is the leader. Note however that the right-hand side is smaller than unity.

${ }^{14}$ Obviously the same occurs in the case of $L$ leader, when $\frac{1+2 d^{2}}{\pi_{L}+\pi_{L} d^{2}+2 d^{2}}<1$ implies that the relative restriction desired by $L$ is sufficiently high.

${ }^{15}$ This outcome is consistent with the comparative statics results presented in the previous section, where we have showed that when both contributions are positive $\frac{\partial \tilde{g}_{C}}{\partial \pi}>0$ and $\frac{\partial \tilde{g}_{B}}{\partial \pi}<0$.

${ }^{16}$ Besides, according to our efficiency analyis, as far as border countries are a minority of the federation, increasing the contribution of central countries can increase social welfare.

\section{Appendix}

\section{Proof of Proposition 1}

In the simultaneous game there is joint contribution when

$$
\frac{d^{2}}{\pi+d^{2}}<\frac{\Delta_{C}}{\Delta_{B}}<\frac{1+d^{2}}{d^{2}}
$$

In the sequential game when $C$ is the leader there is joint contribution when

$$
\frac{d^{2}}{\pi+d^{2}}<\frac{\Delta_{C}}{\Delta_{B}}<\frac{d^{2}+\pi+\pi d^{2}}{d^{2}}
$$

since $\frac{1+d^{2}}{d^{2}}<\frac{d^{2}+\pi+\pi d^{2}}{d^{2}}$, it follows that the interval of $\frac{\Delta_{C}}{\Delta_{B}}$ under which joint contribution occurs is wider in the sequential game.

In the sequential game when $B$ is the leader there is joint contribution when

$$
\frac{d^{2}}{d^{2}+\pi+\pi d^{2}}<\frac{\Delta_{C}}{\Delta_{B}}<\frac{1+d^{2}}{d^{2}}
$$

since $\frac{d^{2}}{d^{2}+\pi+\pi d^{2}}<\frac{d^{2}}{\pi+d^{2}}$, it follows that the interval of $\frac{\Delta_{C}}{\Delta_{B}}$ under which joint contribution occurs is wider in the sequential game. 


\section{Proof of Proposition 2}

We want to prove that total contribution in the simultaneous framework $\left(\tilde{g}_{C}+\tilde{g}_{B}\right)$ dominates total contribution in the sequential framework $\left(g_{C}^{*}+g_{B}^{*}\right.$ and $\left.g_{C}^{* *}+g_{B}^{* *}\right)$. Thus, we have to verify that

$$
\underbrace{\frac{d\left(\pi \Delta_{C}+\Delta_{B}\right)}{\pi+d^{2}+\pi d^{2}}}_{\text {simultaneous }}>\underbrace{\frac{d\left(\pi^{2} \Delta_{C}+\Delta_{B}\left(\pi+d^{2}\right)\right)}{\pi^{2} d^{2}+\left(\pi+d^{2}\right)^{2}}}_{\text {sequential, } C \text { leader }}
$$

Condition (24) boils down to

$$
\frac{\Delta_{C}}{\Delta_{B}}>\frac{d^{2}}{\pi+d^{2}} \text {. }
$$

When $B$ is the leader we have.

$$
\underbrace{\frac{d\left(\pi \Delta_{C}+\Delta_{B}\right)}{\pi+d^{2}+\pi d^{2}}}_{\text {simultaneous }}>\underbrace{\frac{d \Delta_{C}\left(\pi+\pi d^{2}\right)+d \Delta_{B}}{d^{2}+\pi\left(1+d^{2}\right)^{2}}}_{\text {sequential, } B \text { leader }}
$$

which boils down to

$$
\frac{\Delta_{C}}{\Delta_{B}}<\frac{1+d^{2}}{d^{2}}
$$

we conclude that total contribution in the simultaneous framework dominates total contribution in the sequential framework when $\frac{\Delta_{C}}{\Delta_{B}}>\frac{d^{2}}{\pi+d^{2}}(C$ leader $)$ and $\frac{\Delta_{C}}{\Delta_{B}}<\frac{1+d^{2}}{d^{2}}$ ( $B$ leader). However, these conditions coincide with the values of $\frac{\Delta_{C}}{\Delta_{B}}$ assuring joint contribution in the simultaneous framework. Thus we conclude that when both contributions are positive, total contribution in the simultaneous game dominates total contribution in the sequential game.

\section{Proof of Proposition 3}

We want to prove when the leader contributes more than the follower. When $C$ is the leader, the condition $g_{C}^{*} \geq g_{B}^{*}$ is

$$
\frac{\Delta_{C}\left(\pi+d^{2}\right) \pi d-\pi d^{3} \Delta_{B}}{\pi^{2} d^{2}+\left(\pi+d^{2}\right)^{2}} \geq \frac{\Delta_{B}\left(\pi+d^{2}+\pi d^{2}\right) d-\pi d^{3} \Delta_{C}}{\pi^{2} d^{2}+\left(\pi+d^{2}\right)^{2}}
$$

by rearranging condition (26) we obtain

$$
\frac{\Delta_{C}}{\Delta_{B}} \geq \frac{\pi+d^{2}+2 \pi d^{2}}{\pi+2 \pi d^{2}} .
$$

With $B$ leader, we set $g_{B}^{* *} \geq g_{C}^{* *}$ :

$$
\frac{\Delta_{B}\left(1+d^{2}\right) d-d^{3} \Delta_{C}}{d^{2}+\pi\left(1+d^{2}\right)^{2}} \geq \frac{\Delta_{C}\left(d^{2}+\pi+\pi d^{2}\right) d-d^{3} \Delta_{B}}{d^{2}+\pi\left(1+d^{2}\right)^{2}} .
$$

By rearranging condition (27) we obtain

$$
\frac{\Delta_{C}}{\Delta_{B}} \leq \frac{1+2 d^{2}}{\pi+\pi d^{2}+2 d^{2}} .
$$

Since

$$
\frac{d^{2}}{\pi+d^{2}}<\frac{\pi+d^{2}+2 \pi d^{2}}{\pi+2 \pi d^{2}}<\frac{\pi d^{2}+d^{2}+\pi}{\pi d^{2}}
$$

and 


$$
\frac{d^{2}}{d^{2}+\pi+\pi d^{2}}<\frac{1+2 d^{2}}{\pi+\pi d^{2}+2 d^{2}}<\frac{1+d^{2}}{d^{2}}
$$

we conclude that proposition 3 holds when there is joint contribution.

\section{Proof of Proposition 4}

To compare the individual contributions in the simultaneous game, we set $\tilde{g}_{C} \geq \tilde{g}_{B}$, i.e.

$$
\frac{\Delta_{C}\left(\pi+d^{2}\right) d-d^{3} \Delta_{B}}{d^{2}+\pi+\pi d^{2}} \geq \frac{\Delta_{B}\left(1+d^{2}\right) d-d^{3} \Delta_{C}}{d^{2}+\pi+\pi d^{2}} .
$$

By rearranging condition (28) we obtain

$$
\frac{\Delta_{C}}{\Delta_{B}} \geq \frac{\pi+2 d^{2}}{1+2 d^{2}} .
$$

\section{The effect of $\pi$}

$$
\begin{aligned}
& \frac{\partial \tilde{g}_{C}}{\partial \pi}=\frac{d \Delta_{C}\left(d^{2}+\pi+\pi d^{2}\right)-\left(1+d^{2}\right)\left(\Delta_{C}\left(\pi+d^{2}\right) d-d^{3} \Delta_{B}\right)}{\left(d^{2}+\pi+\pi d^{2}\right)^{2}} \\
& \frac{\partial \tilde{g}_{B}}{\partial \pi}=\frac{d^{5} \Delta_{C}\left(d^{2}+2 \pi\right)+2 d^{3} \pi \Delta_{B}\left(\pi d^{2}+\pi+d^{2}\right)}{\left(d^{2}+\pi+\pi d^{2}\right)^{2}}
\end{aligned}
$$

proof that $\frac{\partial g_{C}^{*}}{\partial \pi}<0$ :

$$
\frac{\partial g_{B}^{*}}{\partial \pi}<0 \quad \text { for } \quad \frac{\Delta_{C}}{\Delta_{B}} \leq \frac{\left(\pi+d^{2}+\pi d^{2}\right)^{2}-d^{6}}{\pi^{2} d^{2}\left(1+d^{2}\right)-d^{6}}
$$

but for both contributions to be positive we need $\frac{\Delta_{C}}{\Delta_{B}} \leq \frac{\left(\pi+d^{2}+\pi d^{2}\right)}{\pi d^{2}}$. Since

$$
\frac{\left(\pi+d^{2}+\pi d^{2}\right)}{\pi d^{2}}<\frac{\left(\pi+d^{2}+\pi d^{2}\right)^{2}-d^{6}}{\pi^{2} d^{2}\left(1+d^{2}\right)-d^{6}}
$$

we conclude that $\frac{\partial g_{C}^{*}}{\partial \pi}<0$ when both contributions are positive.

\section{Proof of Proposition 5}

Consider the social welfare function (20):

$$
W=-\frac{\alpha}{2}\left[\left(M-M_{C}^{*}\right)^{2}+g_{C}^{2}\right]-\frac{(1-\alpha)}{2}\left[\left(M-M_{B}^{*}\right)^{2}+\pi g_{B}^{2}\right] .
$$

The total differential of (20) with respect to $g_{C}$ and $g_{B}$ is

$$
\begin{aligned}
d W & =-\frac{\alpha}{2}\left[-2 d\left(\Delta_{C}-d\left(g_{C}+g_{B}\right)\right)\right]\left(d g_{C}+d g_{B}\right)+ \\
& -\frac{(1-\alpha)}{2}\left[-2 d\left(\Delta_{B}-d\left(g_{C}+g_{B}\right)\right)\right]\left(d g_{C}+d g_{B}\right)-\left[\alpha g_{C} d g_{C}+(1-\alpha) \pi g_{B} d g_{B}\right]
\end{aligned}
$$

Suppose now that total contribution $\left(g_{C}+g_{B}\right)$ is kept constant, while some contribution is reallocated between $C$ and $B$. In such a case we have

$$
d g_{C}+d g_{B}=0
$$

by substituting $d g_{B}=-d g_{C}$ the differential $d W$ boils down to

$$
d W=d g_{C}\left[(1-\alpha) \pi g_{B}-\alpha g_{C}\right] .
$$

When $\left[(1-\alpha) \pi g_{B}>\alpha g_{C}\right]$, a reallocation $d g_{C}>0$ increases the social welfare. When $\left[(1-\alpha) \pi g_{B}<\alpha g_{C}\right]$, a reallocation $d g_{C}<0$ increases the social welfare. 
Equality of the weighted marginal costs implies that the more populated country has to increase its contribution because it benefits more from immigration restriction.

In other words, efficiency implies

$$
(1-\alpha) \pi g_{B}^{*}=\alpha g_{C}^{*} .
$$

Since the players do not condider the weight $\alpha$, there is no reason why the decentralized equilibrium should produce such an outcome. We conclude that the equilibrium is generally inefficient.

A particular case arises when $\alpha=1 / 2$, i.e. when $C$ and $B$ are equal in size. In such a case, Pareto efficiency requires the equality of the marginal costs and, to to prove inefficiency, we only have to compare these costs in equilibrium. In the sequential game with $C$ leader we have to set $\left(\pi g_{B}^{*}-g_{C}^{*}\right)>0$, i. e.

$$
\pi\left(\frac{\Delta_{B}\left(\pi+d^{2}+\pi d^{2}\right) d-\pi d^{3} \Delta_{C}}{\pi^{2} d^{2}+\left(\pi+d^{2}\right)^{2}}\right)>\frac{\Delta_{C}\left(\pi+d^{2}\right) \pi d-\pi d^{3} \Delta_{B}}{\pi^{2} d^{2}+\left(\pi+d^{2}\right)^{2}}
$$

which reduces to

$$
\frac{\Delta_{C}}{\Delta_{B}}<\frac{\pi+\pi d^{2}+2 d^{2}}{\pi+d^{2}+\pi d^{2}}
$$

since joint contribution occurs in the interval

$$
\frac{d^{2}}{d^{2}+\pi}<\frac{\Delta_{C}}{\Delta_{B}}<\frac{d^{2}+\pi+\pi d^{2}}{\pi d^{2}}
$$

and since

$$
\frac{d^{2}}{d^{2}+\pi+\pi d^{2}}<\frac{\pi+\pi d^{2}+2 d^{2}}{\pi+d^{2}+\pi d^{2}}<\frac{d^{2}+\pi+\pi d^{2}}{d^{2}}
$$

we conclude that

$$
\begin{array}{ll}
\left(\pi g_{B}^{*}-g_{C}^{*}\right)<0 \quad \text { for } \quad \frac{\Delta_{C}}{\Delta_{B}}>\frac{\pi+\pi d^{2}+2 d^{2}}{\pi+d^{2}+\pi d^{2}} \\
\left(\pi g_{B}^{*}-g_{C}^{*}\right) \geq 0 \quad \text { for } \quad \frac{\Delta_{C}}{\Delta_{B}} \leq \frac{\pi+\pi d^{2}+2 d^{2}}{\pi+d^{2}+\pi d^{2}} .
\end{array}
$$

By applying the same reasoning when $B$ is the leader, the value of $\frac{\Delta_{C}}{\Delta_{B}}$ that equals the marginal costs is

$$
\frac{\Delta_{C}}{\Delta_{B}}=\frac{\pi+d^{2}+\pi d^{2}}{\pi+d^{2}+2 \pi d^{2}}
$$

In this case too, it is easy to check that the critical value of $\frac{\Delta_{C}}{\Delta_{B}}$ lies in the interval of joint contribution:

$$
\frac{d^{2}}{d^{2}+\pi+\pi d^{2}}<\frac{\pi+d^{2}+\pi d^{2}}{\pi+d^{2}+2 \pi d^{2}}<\frac{1+d^{2}}{d^{2}} .
$$

Finally, in the simultaneous game the marginal costs are equalized when

$$
\frac{\Delta_{C}}{\Delta_{B}}=1 .
$$

It is straightforward to verify that 1 lies in the interval of joint contribution:

$$
\frac{d^{2}}{d^{2}+\pi}<1<\frac{1+d^{2}}{d^{2}}
$$




\section{Competing interests}

The IZA Journal of Migration is committed to the IZA Guiding Principles of Research Integrity. The authors declare that they have observed these principles.

\section{Acknowledgments}

We are grateful to an anonymous referee for his/her suggestions. We also thank Vincenzo Pierro, Toke Aidt, David Bartolini, Alberto Bennardo, Gaetano Bloise, Claudio Mezzetti, Annamaria Menichini and Francesco Magris for very helpful comments. Giuseppe Russo acknowledges financial support from the Mobilità Internazionale programme of the Università di Salerno.

Responsible editor: Denis Fougère

\section{Author details}

${ }^{1}$ Dipartimento di Scienze Economiche, Università di Salerno, via Ponte don Melillo, Fisciano (SA), Italy. ${ }^{2}$ CSEF, Napoli, Italy. ${ }^{3}$ Università di Salerno, via Ponte don Melillo, Fisciano (SA), Italy. ${ }^{4}$ University of Leicester, Leicester, England.

Received: 24 February 2013 Accepted: 18 July 2013

Published: 19 August 2013

\section{References}

Amegashie JA (2004) A Political Economy Model of Immigration Quotas, Economics of Governance 5: 255-267 Arrow K (1979) The Property Rights Doctrine and Demand Revelation under Incomplete Information. In: Boskin M (ed) Economics and Human Welfare. Academic Press, New York, pp 23-39

Benhabib J (1996) On the Political Economy of Immigration, European Economic Review 40: 1737-1743

Bergstrom T, Blume, L, Varian, H (1986) On the Private Provision of Pblic Good, Journal of Public Economics 29: 25-49 Clarke EH (1971) Multipart Pricing of Public Goods. Public Choice 11:17 - 33

Cornes R, Sandler T (1984) Easy Riders, Joint Production and Public Goods. Econ J 94: 580-598

D'Aspremont C, Gerard-Varet LA (1979) "Incentives and Incomplete Information". J Public Econ 11: 25-45

Epstein GS, Nitzan S (2006) The Struggle over Migration Policy. J Population Econ 19(4): 703-723

European Commission (2008) Examining the Creation of a European Border Surveillance System (Eurosur) - Impact Assessment SEC(2008): 152. Brussels, 13 February, p. 19

Facchini G, Lorz O, Willmann G (2006) Asylum Seekers in Europe: the Warm Glow of a Hot Potato. J Population Econ 19: $411-430$

Facchini G, Willmann G (2005) The Political Economy of International Factor Mobility. J Int Econ 67: 201-219

Haake CJ, Krieger T, Minter S (2010) "On the Institutional Design of Burden Sharing when Financing External Border Enforcement in the EU". Center for International Economics WP n, Germany

Hatton T (2004) Seeking Asylum in Europe. Econ Policy 38: 5-62

Jeandesboz J (2008) Reinforcing the Surveillance of EU Borders: the Future Developments of Frontex and Eurosur. Challenge Res Paper n, Brussels

Mayr K, Minter S, Krieger T (2011) "Policies on Illegal Immigration in a Federation". Regional Science and Urban Econ 42: $153-165$

Mazza I, Van Winden, F (1996) A Political Economic Analysis of Labor Migration and Income Redistribution. Public Choice 88(3-4): 333-63

Russo G (2011) "Voting over Selective Immigration Policies with Immigration Aversion". Economics of Governance 12(4): 325-351

Russo G, Senatore L (2012) "A Note on Contribution Games with Loss Functions". Econ Lett 115(2): 211-214

Schain MA (2010) "The Shaping of European Immigration Policy during the past Decade". Int J Econ Bus Res 2(1/2): 112-124

Varian H (1994) "Sequential Contributions to Public Goods". J Public Econ 53(2): 165 - 186

Warr PG (1982) "The Private Provision of a Public Good Independent of the Distribution of Income". Econ Lett 13: 207-211

Warr PG (1983) "Pareto Optimal Redistribution and Private Charity". J Public Econ 19: 131-138

Wolff S (2008) "Border Management in the Mediterranean: Internal, External and Ethical Challenges". Cambridge Rev Int Affairs 21(2): 253-271

doi:10.1186/2193-9039-2-16

Cite this article as: Russo and Senatore: Who contributes? A strategic approach to a European immigration policy. IZA Journal of Migration 2013 2:16. 